Alessio Porretta $\cdot$ Laurent Véron

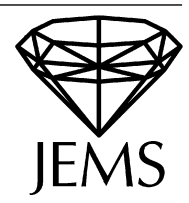

\title{
Separable solutions of quasilinear Lane-Emden equations
}

Received May 25, 2011 and in revised form October 6, 2011

\begin{abstract}
For $0<p-1<q$ and either $\epsilon=1$ or $\epsilon=-1$, we prove the existence of solutions of $-\Delta_{p} u=\epsilon u^{q}$ in a cone $C_{S}$, with vertex 0 and opening $S$, vanishing on $\partial C_{S}$, of the form $u(x)=$ $|x|^{-\beta} \omega(x /|x|)$. The problem reduces to a quasilinear elliptic equation on $S$ and the existence proof is based upon degree theory and homotopy methods. We also obtain a nonexistence result in some critical case by making use of an integral type identity.
\end{abstract}

Keywords. Quasilinear elliptic equations, $p$-Laplacian, cones, Leray-Schauder degree

\section{Introduction}

It is well established that the description of the boundary behavior of positive singular solutions of Lane-Emden equations

$$
-\Delta u=\epsilon u^{q}
$$

with $q>1$ in a domain $\Omega \subset \mathbb{R}^{N}$ is greatly facilitated by using specific separable solutions of this equation. This was shown in 1991 by Gmira-Véron [7] in the case $\epsilon=-1$ and more recently by Bidaut-Véron-Ponce-Véron [3] in the case $\epsilon=1$. If the domain is assumed to be a cone $C_{S}=\left\{x \in \mathbb{R}^{N} \backslash\{0\}: x /|x| \in S\right\}$ with vertex 0 and opening $S \subsetneq S^{N-1}$ (the unit sphere in $\mathbb{R}^{N}$ ), separable solutions of (1.1) vanishing on $\partial C_{S} \backslash\{0\}$ are of the form

$$
u(x)=|x|^{-2 /(q-1)} \omega(x /|x|),
$$

with $\omega$ satisfying

$$
-\Delta^{\prime} \omega-\ell_{q, N} \omega-\epsilon \omega^{q}=0 \quad \text { in } S
$$

A. Porretta: Dipartimento di Matematica, Università di Roma Tor Vergata,

Via della Ricerca Scientifica 1, 00133 Roma, Italy; e-mail: porretta@mat.uniroma2.it

L. Véron: Laboratoire de Mathématiques et Physique Théorique, CNRS UMR 6083, Faculté des Sciences, Parc de Grandmont, Université François Rabelais, Tours 37200, France; e-mail: veronl@univ-tours.fr

Mathematics Subject Classification (2010): 35J92, 35J60, 47H11, 58C30 
vanishing on $\partial S$ and where $\ell_{q, N}=\frac{2}{q-1} \frac{2 q}{q-1}-N$ and $\Delta^{\prime}$ is the Laplace-Beltrami operator on $S^{N-1}$. To this equation is associated the functional

$$
J(\phi):=\int_{S}\left(\frac{1}{2}\left|\nabla^{\prime} \phi\right|^{2}-\frac{\ell_{q, N}}{2} \phi^{2}-\frac{\epsilon}{q+1}|\phi|^{q+1}\right) d v_{g},
$$

where $\nabla^{\prime}$ is the covariant derivative on $S^{N-1}$. In the case $\epsilon=1$, nonexistence of a nontrivial positive solution of (1.3) when $\ell_{q, N} \geq \lambda_{S}$ (the first eigenvalue of $-\Delta^{\prime}$ in $W_{0}^{1,2}(S)$ ) follows by multiplying the equation by the first eigenfunction and integrating over $S$; existence holds when $\ell_{q, N}<\lambda_{S}$ and $q<(N+1) /(N-3)$ by classical variational methods, and again nonexistence holds when $q \geq(N+1) /(N-3)$ and $S \subset S_{+}^{N-1}$ is starshaped by using an integral identity [3, Th. 2.1, Cor. 2.1]. When $\epsilon=-1$, nonexistence of a nontrivial solution of (1.3) when $\ell_{q, N} \leq \lambda_{S}$ is obtained by multiplying the equation by $\omega$ and integrating over $S$, while existence when $\ell_{q, N}>\lambda_{S}$ follows by minimizing $J$ over $W_{0}^{1,2}(S) \cap L^{q+1}(S)$.

In this paper we investigate similar questions for the quasilinear Lane-Emden equations

$$
-\operatorname{div}\left(|\nabla u|^{p-2} \nabla u\right)=\epsilon u^{q} \quad \text { in } C_{S},
$$

where $S$ is a smooth subset of $S^{N-1}, q>p-1>0$ and $\epsilon= \pm 1$, and we look for positive solutions $u$, vanishing on $\partial C_{S} \backslash\{0\}$, of the separable form

$$
u(x)=|x|^{-\beta} \omega(x /|x|) .
$$

It is straightforward to check that $u$ is a solution of (1.5) provided

$$
\beta=\beta_{q}:=\frac{p}{q+1-p}
$$

and $\omega$ is a positive solution of

$$
-\operatorname{div}\left(\left(\beta_{q}^{2} \omega^{2}+\left|\nabla^{\prime} \omega\right|^{2}\right)^{(p-2) / 2} \nabla^{\prime} \omega\right)-\beta_{q} \lambda\left(\beta_{q}\right)\left(\beta_{q}^{2} \omega^{2}+\left|\nabla^{\prime} \omega\right|^{2}\right)^{(p-2) / 2} \omega=\epsilon \omega^{q}
$$

in $S$ vanishing on $\partial S$, where $\operatorname{div}(\cdot)$ is the divergence operator defined according to the intrinsic metric $g$ and where we have set

$$
\lambda(\beta)=\beta(p-1)+p-N .
$$

If $\epsilon=0$, it is now well-known that positive $p$-harmonic functions in $C_{S}$ vanishing on $\partial C_{S}$ exist in the form (1.6), and either they are regular at 0 and $\beta=-\tilde{\beta}_{S}<0$, or they are singular and $\beta=\beta_{S}>0$, where the values of $\tilde{\beta}_{S}, \beta_{S}$ are unique. In this case $\omega=\tilde{\omega}_{S}$ or $\omega_{S}$ is a solution of

$$
-\operatorname{div}\left(\left(\beta^{2} \omega^{2}+\left|\nabla^{\prime} \omega\right|^{2}\right)^{(p-2) / 2} \nabla^{\prime} \omega\right)-\beta \lambda(\beta)\left(\beta^{2} \omega^{2}+\left|\nabla^{\prime} \omega\right|^{2}\right)^{(p-2) / 2} \omega=0
$$

in $S$, where $\beta=\tilde{\beta}_{S}$ or $\beta_{S}$. The existence of $\left(\tilde{\beta}_{S}, \tilde{\omega}_{S}\right)$ is due to Tolksdorf in a pioneering work [18]. Tolksdorf's method has been adapted by Véron [20] in order to prove the existence of $\left(\beta_{S}, \omega_{S}\right)$. Later on Porretta and Véron [13] obtained a more general proof 
of the existence of such couples. Notice that $\beta_{S}$ (as well as $\tilde{\beta}_{S}$ ) is uniquely determined while $\omega$ is unique up to homothety. In both cases the proofs rely on the strong maximum principle.

When $p \neq 2$, existence of a nontrivial solution in the case $\epsilon=1$ is obtained in [2] when $N=2$ and $\beta_{q}<\beta_{S}$ by a dynamical system approach; while if $\epsilon=-1$ and $\beta_{q}>\beta_{S}$, the existence is proved in [20] by a suitable adaptation of Tolksdorf's construction. Notice that no functional can be associated to (1.8), except in the case $q=q^{*}=N p /(N-p)-1$. In that case, (1.8) is the Euler-Lagrange equation for the functional

$$
J_{q}(\phi):=\int_{S}\left(\frac{1}{p}\left(\beta_{q^{*}}^{2} \phi^{2}+\left|\nabla^{\prime} \phi\right|^{2}\right)^{p / 2}-\frac{\epsilon}{q^{*}+1}|\phi|^{q^{*}+1}\right) d v_{g},
$$

and existence of a nontrivial solution of (1.8) with $\epsilon=1$ is derived from the mountain pass theorem. In all the other cases variational techniques cannot be used and have to be replaced by topological methods based upon Leray-Schauder degree. Define $q_{c}$ by

$$
q_{c}=q_{c, p}= \begin{cases}\frac{(N-1) p}{N-1-p}-1 & \text { if } p<N-1 \\ \infty & \text { if } p \geq N-1\end{cases}
$$

Then we prove the following results:

I. Let $\epsilon=1$. Assume $p>1, q<q_{c}$ and $\beta_{q}<\beta_{S}$. Then (1.8) admits a positive solution in $S$ vanishing on $\partial S$.

II. Let $\epsilon=-1$. Assume $p>1$ and $\beta_{q}>\beta_{S}$. Then (1.8) admits a unique positive solution in $S$ vanishing on $\partial S$.

The result I is based upon sharp Liouville theorems for solutions of (1.5) in $\mathbb{R}^{N}$ or $\mathbb{R}_{+}^{N}$ respectively due to Serrin-Zou [17] and Zou [23]. In the case of II, the existence part is already known, but we give here a simpler form than the one in [20], using a topological deformation acting on the exponent $p$. In the case $\epsilon=1$, the result is optimal in the case $q=q_{c}$; indeed, using an integral identity, we also prove

III. Let $\epsilon=1, S \subsetneq S_{+}^{N-1}$ be a starshaped domain and $1<p<N-1$. If $q=q_{c}$, then (1.8) admits no positive solution in $S$ vanishing on $\partial S$.

Notice that when $p=2$ an integral identity was used in [3] to prove nonexistence for all $q \geq q_{c, 2}$. The form which is derived in the case $p \neq 2$ is much more complicated and we prove nonexistence only in the case $q=q_{c, p}$.

Finally, the constraint $\beta_{q}<\beta_{S}$ in I (respectively, $\beta_{q}>\beta_{S}$ in II) is sharp. When $\epsilon=1$, the nonexistence of positive solutions of (1.8) when $\beta_{q} \geq \beta_{S}$ has been proved in [2]. The method is based upon the strong maximum principle. When $\epsilon=-1$ a somewhat similar method is used in [22] and yields nonexistence results when $\beta_{q} \leq \beta_{S}$. Notice that obtaining such results when $p=2$ is straightforward. 


\section{Nonexistence for the reaction problem}

Let $S$ be a bounded $C^{2}$ subdomain of $S^{N-1}$. We consider positive solutions in $S$ of

$$
-\operatorname{div}\left(\left(\beta^{2} \omega^{2}+\left|\nabla^{\prime} \omega\right|^{2}\right)^{(p-2) / 2} \nabla^{\prime} \omega\right)-\beta \lambda(\beta)\left(\beta^{2} \omega^{2}+\left|\nabla^{\prime} \omega\right|^{2}\right)^{(p-2) / 2} \omega=\omega^{q}
$$

vanishing on $\partial S$. Recall that $\lambda(\beta)$ is given by (1.9) and that, in connection with problem (1.5), we are interested in the special case where $\beta=\beta_{q}$ is given by (1.7). The following Pohozaev type identity, which is valid for any $\beta$, is the key to nonexistence. We denote by $S_{+}^{N-1}$ the half-sphere.

Proposition 2.1. Let $S \subsetneq S^{N-1}$ be a $C^{2}$ domain and $\phi$ the first eigenfunction of $-\Delta^{\prime}$ in $W_{0}^{1,2}\left(S_{+}^{N-1}\right)$. If $\omega \in W_{0}^{1, p}(S) \cap C(\bar{S})$ is a positive solution of $(2.1)$ in $S$, and if we set $\Omega=\left(\beta^{2} \omega^{2}+\left|\nabla^{\prime} \omega\right|^{2}\right)^{1 / 2}$, then

$\left(1-\frac{1}{p}\right) \int_{\partial S}\left|\omega_{\nu}\right|^{p} \phi_{\nu} d S=A \int_{S} \omega^{q+1} \phi d \sigma+B \int_{S} \Omega^{p-2}\left|\nabla^{\prime} \omega\right|^{2} \phi d \sigma+C \int_{S} \Omega^{p-2} \omega^{2} \phi d \sigma$

with

$$
\begin{aligned}
& A=A(\beta):=-\frac{N-1}{q+1}-\beta(p \beta+p-N), \\
& B=B(\beta):=\frac{N-1-p}{p}+\beta(p \beta+p-N), \\
& C=C(\beta):=\beta^{2}\left(\frac{N-1}{p}-(p \beta+p-N) \lambda(\beta)\right) .
\end{aligned}
$$

In order to prove Proposition 2.1, we start with the following lemma.

Lemma 2.1. Let $S \subset S^{N-1}$ be a $C^{2}$ domain and $\phi \in C^{2}(\bar{S})$. If $\omega \in W_{0}^{1, p}(S) \cap C(\bar{S})$ is a positive solution of (2.1) in $S$, then

$$
\begin{gathered}
\left(1-\frac{1}{p}\right) \int_{\partial S}\left|\omega_{\nu}\right|^{p} \phi_{\nu} d S=\int_{S}\left(\frac{\Delta^{\prime} \phi}{q+1}-\beta(p \beta+p-N) \phi\right) \omega^{q+1} d \sigma-\frac{1}{p} \int_{S} \Omega^{p} \Delta^{\prime} \phi d \sigma \\
+\int_{S} \Omega^{p-2} D^{2} \phi\left(\nabla^{\prime} \omega, \nabla^{\prime} \omega\right) d \sigma+\beta(p \beta+p-N) \int_{S} \Omega^{p-2}\left|\nabla^{\prime} \omega\right|^{2} \phi d \sigma \\
-\beta^{2}(p \beta+p-N) \lambda(\beta) \int_{S} \Omega^{p-2} \omega^{2} \phi d \sigma
\end{gathered}
$$

Proof. By the regularity theory of $p$-Laplace type equations (see e.g. [6], [19] and Appendix in [13]) it turns out that $\omega \in C^{1, \gamma}(\bar{S})$ for some $\gamma \in(0,1)$, and since $\beta^{2} \omega^{2}+$ $\left|\nabla^{\prime} \omega\right|^{2}>0$ in the interior, by elliptic regularity we have $\omega \in C^{2}(S)$. Let $\phi \in C^{2}(S)$ be a given function and $\zeta \in C_{c}^{1}(S)$; since $\zeta$ is compactly supported we can multiply 
(2.1) by the test function $\left\langle\nabla^{\prime} \omega, \nabla^{\prime} \phi\right\rangle \zeta$. Integrating by parts we get (using the notation $\left.\Omega:=\left(\beta^{2} \omega^{2}+\left|\nabla^{\prime} \omega\right|^{2}\right)^{1 / 2}\right)$

$$
\begin{aligned}
\int_{S} \Omega^{p-2}\left(\frac{1}{2}\left\langle\nabla^{\prime}\left|\nabla^{\prime} \omega\right|^{2}, \nabla^{\prime} \phi\right\rangle+D^{2} \phi\left(\nabla^{\prime} \omega,\right.\right. & \left.\left.\nabla^{\prime} \omega\right)\right) \zeta d \sigma \\
& +\int_{S} \Omega^{p-2}\left\langle\nabla^{\prime} \omega, \nabla \zeta\right\rangle\left\langle\nabla^{\prime} \omega, \nabla^{\prime} \phi\right\rangle d \sigma \\
=\beta \lambda(\beta) \int_{S} \Omega^{p-2} \omega\left\langle\nabla^{\prime} \omega, \nabla^{\prime} \phi\right\rangle \zeta d \sigma & +\frac{1}{q+1} \int_{S}\left\langle\nabla^{\prime} \omega^{q+1}, \nabla^{\prime} \phi\right\rangle \zeta d \sigma .
\end{aligned}
$$

Since

$$
\Omega^{p-2} \frac{1}{2}\left\langle\nabla^{\prime}\left|\nabla^{\prime} \omega\right|^{2}, \nabla^{\prime} \phi\right\rangle=\frac{1}{p}\left\langle\nabla^{\prime} \Omega^{p}, \nabla \phi\right\rangle-\beta^{2} \Omega^{p-2} \omega\left\langle\nabla^{\prime} \omega, \nabla^{\prime} \phi\right\rangle
$$

we obtain, due to (1.9),

$$
\begin{aligned}
& \frac{1}{p} \int_{S}\left\langle\nabla^{\prime} \Omega^{p}, \nabla^{\prime} \phi\right\rangle \zeta d \sigma+\int_{S} \Omega^{p-2} D^{2} \phi\left(\nabla^{\prime} \omega, \nabla^{\prime} \omega\right) \zeta d \sigma \\
& \quad+\int_{S} \Omega^{p-2}\left\langle\nabla^{\prime} \omega, \nabla^{\prime} \zeta\right\rangle\left\langle\nabla^{\prime} \omega, \nabla^{\prime} \phi\right\rangle d \sigma \\
& =\beta(p \beta+p-N) \int_{S} \Omega^{p-2} \omega\left\langle\nabla^{\prime} \omega, \nabla^{\prime} \phi\right\rangle \zeta d \sigma+\frac{1}{q+1} \int_{S}\left\langle\nabla^{\prime} \omega^{q+1}, \nabla^{\prime} \phi\right\rangle \zeta d \sigma .
\end{aligned}
$$

Integrating by parts the first and the last terms we get

$$
\begin{array}{r}
-\frac{1}{p} \int_{S} \Omega^{p}\left\langle\nabla^{\prime} \phi, \nabla^{\prime} \zeta\right\rangle d \sigma+\frac{1}{q+1} \int_{S} \omega^{q+1}\left\langle\nabla^{\prime} \phi, \nabla^{\prime} \zeta\right\rangle d \sigma+\int_{S}\left(\frac{\omega^{q+1}}{q+1}-\frac{\Omega^{p}}{p}\right) \Delta^{\prime} \phi \zeta d \sigma \\
+\int_{S} \Omega^{p-2} D^{2} \phi\left(\nabla^{\prime} \omega, \nabla^{\prime} \omega\right) \zeta d \sigma+\int_{S} \Omega^{p-2}\left\langle\nabla^{\prime} \omega, \nabla^{\prime} \zeta\right\rangle\left\langle\nabla^{\prime} \omega, \nabla^{\prime} \phi\right\rangle d \sigma \\
=\beta(p \beta+p-N) \int_{S} \Omega^{p-2} \omega\left\langle\nabla^{\prime} \omega, \nabla^{\prime} \phi\right\rangle \zeta d \sigma
\end{array}
$$

Now we choose $\zeta=\zeta_{\delta}$, where $\zeta_{\delta}$ is a sequence of compactly supported $C^{1}$ functions such that $\zeta_{\delta}(\sigma) \rightarrow 1$ for every $\sigma \in S$ and $\left|\nabla^{\prime} \zeta_{\delta}\right|$ is bounded in $L^{1}(S)$. It is easy to see by integration by parts that for every continuous vector field $F \in C(\bar{S})$ we have

$$
\int_{S}\left\langle F, \nabla^{\prime} \zeta_{\delta}\right\rangle d \sigma \rightarrow-\int_{\partial S}\langle F, v(\sigma)\rangle d \sigma
$$

where $v$ is the outward unit normal on $\partial S$. We take $\zeta=\zeta_{\delta}$ in (2.7) and we let $\delta \rightarrow 0$. Using that $\omega \in C^{1}(\bar{S})$ and that, by the Hopf lemma, $\omega_{\nu}:=\left\langle\nabla^{\prime} \omega, \nu(\sigma)\right\rangle<0$ we can actually pass to the limit in the integrals containing $\nabla^{\prime} \zeta_{\delta}$. Recalling that $\omega=0$ and $\nabla^{\prime} \omega=-\left|\omega_{\nu}\right| \nu$ on $\partial S$ we obtain

$$
\begin{aligned}
\left(1-\frac{1}{p}\right) \int_{\partial S}\left|\omega_{\nu}\right|^{p} \phi_{\nu} d S= & \int_{S}\left(\frac{\omega^{q+1}}{q+1}-\frac{\Omega^{p}}{p}\right) \Delta^{\prime} \phi d \sigma+\int_{S} \Omega^{p-2} D^{2} \phi\left(\nabla^{\prime} \omega, \nabla^{\prime} \omega\right) d \sigma \\
& -\beta(p \beta+p-N) \int_{S} \Omega^{p-2} \omega\left\langle\nabla^{\prime} \omega, \nabla^{\prime} \phi\right\rangle d \sigma .
\end{aligned}
$$


Multiplying (2.1) by $\omega \phi$ we derive

$$
\begin{aligned}
\int_{S} \Omega^{p-2} \omega\left\langle\nabla^{\prime}\right. & \left.\omega, \nabla^{\prime} \phi\right\rangle d \sigma \\
& =-\int_{S} \Omega^{p-2}\left|\nabla^{\prime} \omega\right|^{2} \phi d \sigma+\beta \lambda(\beta) \int_{S} \Omega^{p-2} \omega^{2} \phi d \sigma+\int_{S} \omega^{q+1} \phi d \sigma
\end{aligned}
$$

so that (2.8) becomes, replacing its last term,

$$
\begin{gathered}
\left(1-\frac{1}{p}\right) \int_{\partial S}\left|\omega_{\nu}\right|^{p} \phi_{\nu} d S=\int_{S}\left(\frac{\omega^{q+1}}{q+1}-\frac{\Omega^{p}}{p}\right) \Delta^{\prime} \phi d \sigma+\int_{S} \Omega^{p-2} D^{2} \phi\left(\nabla^{\prime} \omega, \nabla^{\prime} \omega\right) d \sigma \\
-\beta(p \beta+p-N) \int_{S} \omega^{q+1} \phi d \sigma+\beta(p \beta+p-N) \int_{S} \Omega^{p-2}\left|\nabla^{\prime} \omega\right|^{2} \phi d \sigma \\
-\beta^{2}(p \beta+p-N) \lambda(\beta) \int_{S} \Omega^{p-2} \omega^{2} \phi d \sigma
\end{gathered}
$$

which is (2.6).

Proof of Proposition 2.1. We use Lemma 2.1 choosing in (2.6) $\phi$ to be the first eigenfunction of $-\Delta^{\prime}$ in $W_{0}^{1,2}\left(S_{+}^{N-1}\right)$. Since $\Delta^{\prime} \phi=(1-N) \phi$ and $D^{2} \phi=-\phi g_{0}$, we get

$$
\begin{aligned}
\left(1-\frac{1}{p}\right) \int_{\partial S}\left|\omega_{\nu}\right|^{p} \phi_{\nu} d S= & -\int_{S}\left(\frac{N-1}{q+1}+\beta(p \beta+p-N)\right) \omega^{q+1} \phi d \sigma \\
& +\frac{N-1}{p} \int_{S} \Omega^{p} \phi d \sigma-\int_{S} \Omega^{p-2}\left|\nabla^{\prime} \omega\right|^{2} \phi d \sigma \\
& +\beta(p \beta+p-N) \int_{S} \Omega^{p-2}\left|\nabla^{\prime} \omega\right|^{2} \phi d \sigma \\
& -\beta^{2}(p \beta+p-N) \lambda(\beta) \int_{S} \Omega^{p-2} \omega^{2} \phi d \sigma .
\end{aligned}
$$

Then, using also the definition of $\Omega$, (2.2) follows, with $A, B$ and $C$ given by (2.3)-(2.5).

We shall say that a $C^{2}$ domain $S \subset S_{+}^{N-1}$ is starshaped if there exists a spherical harmonic $\phi$ of degree 1 such that $\phi>0$ on $S$ and for any $a \in \partial S$,

$$
\left\langle\nabla \phi, v_{a}\right\rangle \leq 0
$$

where $v_{a}$ is the unit outward normal vector to $\partial S$ at $a$ in the tangent plane $T_{a}$ to $S^{N-1}$. It also means that there exists some $x_{0} \in S$ such that the geodesic connecting $x_{0}$ and $a$ remains inside $S$.

Theorem 2.1. Assume that $1<p<N-1, q=q_{c}$ and $S \subset S_{+}^{N-1}$ is starshaped. Then (2.1) admits no positive solution in $S$ vanishing on $\partial S$. 
Proof. Recall that in (1.8) we have $\beta_{q}=p /(q-(p-1))$, hence different values of $q$ are in one-to-one correspondence with different values of $\beta$. We first notice that if $q=q_{c}$ then the corresponding critical $\beta$ is given by

$$
\beta_{c}:=\frac{p}{q_{c}-(p-1)}=\frac{N-1-p}{p} .
$$

We now use Proposition 2.1 with $\beta=\beta_{q}$ and we analyze the values of the coefficients $A$, $B, C$ given by (2.3)-(2.5) as functions of $\beta$. First of all, since $q+1=p(1+\beta) / \beta$, we have

$$
A=-\frac{(N-1) \beta}{p(1+\beta)}-\beta(p \beta+p-N)=-\frac{\beta}{\beta+1}\left(\frac{N-1}{p}+p(\beta+1)^{2}-N(\beta+1)\right),
$$

and since from (2.11) we have $\beta_{c}+1=\frac{N-1}{p}$, we deduce

$$
A=-\frac{\beta}{\beta+1} p\left(\beta+1-\frac{1}{p}\right)\left(\beta-\beta_{c}\right) .
$$

Still using (2.11), we also get

$$
B=\beta_{c}+\beta\left(p\left(\beta-\beta_{c}\right)-1\right)=\left(\beta-\beta_{c}\right)(\beta p-1) .
$$

Finally, using (1.9) and (2.11) we have

$$
\begin{aligned}
C & =\beta^{2}\left(\frac{N-1}{p}-(p \beta+p-N)((p-1) \beta+p-N)\right) \\
& =\beta^{2}\left(\beta_{c}+1-\left(p\left(\beta-\beta_{c}\right)-1\right)\left(p\left(\beta-\beta_{c}\right)-(\beta+1)\right)\right) \\
& =\beta^{2}\left(\beta-\beta_{c}\right)(1-p)\left(p \beta-1-\frac{p(N-p)}{p-1}\right) .
\end{aligned}
$$

Therefore $A \geq 0, B \geq 0$ and $C \geq 0$ can be obtained only if $q=q_{c}$, i.e. $\beta=\beta_{c}$, in which case $A=B=C=0$. Since $\phi_{v} \leq 0$ because $S$ is starshaped, we deduce from (2.2) that $\left|\omega_{\nu}\right|^{p} \phi_{\nu}=0$ on $\partial S$. Unless $\omega$ is identically zero, we have $\omega_{\nu}<0$ by the Hopf lemma. Then $\phi_{\nu} \equiv 0$, and using the equation satisfied by $\phi$ and the Gauss formula, we derive

$$
\lambda_{S} \int_{S} \phi d \sigma=0, \quad \text { so } \quad \phi \equiv 0 \text { in } S
$$

which is impossible since $\phi>0$ in $S_{+}^{N-1}$. This proves the first assertion.

Remark. If $p=2$, it is proved in [3] that the nonexistence result of Theorem 2.1 holds for every $q \geq q_{c}$, which suggests that our result above is not optimal. The proof in [3] cannot be applied here since the term $\int_{S} \Omega^{p-2} \omega\left\langle\nabla^{\prime} \omega, \nabla^{\prime} \phi\right\rangle d \sigma$ is completely integrable only if $p=2$. However, we conjecture that, even when $p \neq 2$, the conclusion of Theorem 2.1 holds under the more general condition $q \geq q_{c}$. 
Remark. If we assume that $p \neq 2$, the proof of Theorem 2.1 relies on the existence of a positive function $\phi$ in $S$, satisfying (2.10) on $\partial S$ and

$$
\begin{aligned}
& \frac{\Delta^{\prime} \phi}{(q+1) \phi}-\beta(p \beta+p-N) \geq 0, \\
& \frac{p D^{2} \phi(\xi, \xi)-\Delta^{\prime} \phi}{p \phi}+\beta(p \beta+p-N) \geq 0 \quad \forall \xi \in S^{N-1}, \\
& -\frac{\Delta^{\prime} \phi}{p \phi}-(p \beta+p-N)((p-1) \beta+p-N) \geq 0 .
\end{aligned}
$$

Remark 2.1. For completeness, we recall the nonexistence result obtained in [2, Th. 1]:

Let $\epsilon=1$ and $0<p-1<q$. If $\beta_{q} \geq \beta_{S}$, then there exists no positive solution of (1.8) in $S$ which vanishes on $\partial S$.

\section{Existence for the reaction problem}

Concerning the problem with reaction we consider a more general statement than Theorem I, replacing the sphere by a complete $d$-dimensional Riemannian manifold $(M, g)$ and supposing that $S$ is a relatively compact smooth open domain of $M$. We denote by $\nabla:=\nabla_{g}$ the gradient of a function identified with its covariant derivatives, and by $\operatorname{div}:=\operatorname{div}_{g}$ the intrinsic divergence operator acting on vector fields. The following result is proved in [13].

Theorem 3.1. For any $\beta>0$ there exists a unique $\Lambda_{\beta}>0$ and a unique (up to homothety) positive function $\omega_{\beta} \in C^{2}(S) \cap C^{1}(\bar{S})$ satisfying

$\left\{\begin{array}{l}-\operatorname{div}\left(\left(\beta^{2} \omega_{\beta}^{2}+\left|\nabla \omega_{\beta}\right|^{2}\right)^{(p-2) / 2} \nabla \omega_{\beta}\right)=\beta \Lambda_{\beta}\left(\beta^{2} \omega_{\beta}^{2}+\left|\nabla \omega_{\beta}\right|^{2}\right)^{(p-2) / 2} \omega_{\beta} \quad \text { in } S, \\ \omega_{\beta}=0 \text { on } \partial S .\end{array}\right.$

The mapping $\beta \mapsto \Lambda_{\beta}$ is continuous and decreasing, and the spectral exponent $\beta_{S}$ is the unique $\beta>0$ such that $\Lambda_{\beta_{S}}=\beta_{S}(p-1)+p-d-1$.

Remark 3.1. Let us notice that the monotonicity character of $\beta \mapsto \Lambda_{\beta}$ implies that

$$
0<\beta<\beta_{S} \Leftrightarrow \Lambda_{\beta}-\beta(p-1)>\Lambda_{\beta_{S}}-\beta_{S}(p-1)=p-d-1 .
$$

Therefore, if we set $\lambda(\beta)=\beta(p-1)+p-d-1$, we deduce that

$$
0<\beta<\beta_{S} \Leftrightarrow \Lambda_{\beta}>\lambda(\beta) .
$$

Let us now prove the existence of solutions for the reaction problem.

Theorem 3.2. Assume $1<p<d$ and $p-1<q<q_{c}:=p d /(d-p)-1$. Then for any $0<\beta<\beta_{S}$, there exists a positive function $\omega \in C(\bar{S}) \cap C^{2}(S)$ satisfying

$$
\left\{\begin{array}{l}
-\operatorname{div}\left(\left(\beta^{2} \omega^{2}+|\nabla \omega|^{2}\right)^{p / 2-1} \nabla \omega\right)=\beta \lambda(\beta)\left(\beta^{2} \omega^{2}+|\nabla \omega|^{2}\right)^{p / 2-1} \omega+\omega^{q} \quad \text { in } S, \\
\omega=0 \text { on } \partial S,
\end{array}\right.
$$

where $\lambda(\beta)=\beta(p-1)+p-d-1$. 
In order to prove Theorem 3.2, we use topological arguments as is often needed in a nonvariational setting. In particular, following a strategy similar to [15], our proof is based upon the following fixed point theorem which is a consequence of the Leray-Schauder degree theory to compute the fixed point index of compact mappings. Such results were developed mostly by Krasnosel'skii [9]; we refer to Proposition 2.1 and Remark 2.1 in [5] for the statement below.

Theorem 3.3. Let $X$ be a Banach space and $K \subset X$ a closed cone with non-empty interior. Let $F: K \times \mathbb{R}_{+} \rightarrow K$ be a compact mapping, and let $\Phi(u)=F(u, 0)$ (a compact mapping from $K$ into $K$ ). Assume that there exist $R_{1}<R_{2}$ and $T>0$ such that

(i) $u \neq s \Phi(u)$ for every $s \in[0,1]$ and every $u$ with $\|u\|=R_{1}$.

(ii) $F(u, t) \neq u$ for every $(u, t)$ with $\|u\| \leq R_{2}$ and $t \geq T$.

(iii) $F(u, t) \neq u$ for every $u$ with $\|u\|=R_{2}$ and every $t \geq 0$.

Then the mapping $\Phi$ has a fixed point $u$ such that $R_{1}<\|u\|<R_{2}$.

We also recall the following nonexistence results respectively due to Serrin and Zou [17] and Zou [23].

Theorem 3.4. Assume $1<p<d$ and $p-1<q<q_{c}$. Then there exists no positive $C^{1}$ solution of

$$
-\Delta_{p} u=u^{q}
$$

in $\mathbb{R}^{d}$.

Theorem 3.5. Assume $1<p<d$ and $p-1<q<q_{c}$. Then there exists no positive $C^{1}$ solution of

$$
-\Delta_{p} u=u^{q}
$$

in $\mathbb{R}_{+}^{d}:=\left\{x=\left(x_{1}, \ldots, x_{d}\right): x_{d}>0\right\}$ vanishing on $\partial \mathbb{R}_{+}^{d}:=\left\{x=\left(x_{1}, \ldots, x_{d}\right)\right.$ : $\left.x_{d}=0\right\}$.

Proof of Theorem 3.2. Define the operator $\mathcal{A}$ in $W_{0}^{1, p}(S)$ as

$$
\mathcal{A}(\omega):=-\operatorname{div}_{g}\left(\left(\beta^{2} \omega^{2}+|\nabla \omega|^{2}\right)^{p / 2-1} \nabla \omega\right)+\beta^{2} \omega\left(\beta^{2} \omega^{2}+|\nabla \omega|^{2}\right)^{p / 2-1} .
$$

Note that $\mathcal{A}$ is the derivative of the functional

$$
J(w)=\frac{1}{p} \int_{S}\left(\beta^{2} \omega^{2}+|\nabla \omega|^{2}\right)^{p / 2} d v_{g}
$$

Since $J$ is strictly convex, $\mathcal{A}$ is a strictly monotone operator from $W_{0}^{1, p}(S)$ into $W^{-1, p^{\prime}}(S)$, hence its inverse is well defined and continuous [12]. In order to apply Theorem 3.3, we denote by $X=C_{0}^{1}(\bar{S})$ the closure of $C_{0}^{1}(S)$ in $C^{1}(\bar{S})$. Clearly $X \subset W_{0}^{1, p}(S)$, with continuous imbedding, if it is endowed with its natural norm $\|\cdot\|_{X}:=\|\cdot\|_{C^{1}(\bar{S})}$. Furthermore, since $\partial S$ is $C^{2}, C^{1}(\bar{S}) \cap W_{0}^{1, p}(S)=C_{0}^{1}(\bar{S})$. If $K$ is the cone of nonnegative functions in $S$, it has a nonempty interior. For $t>0$, we set

$$
F(\omega, t):=\mathcal{A}^{-1}\left(\beta(\lambda(\beta)+\beta+t) \omega\left(\beta^{2} \omega^{2}+|\nabla \omega|^{2}\right)^{p / 2-1}+(\omega+t)^{q}\right) .
$$


Note that

$$
\Phi(\omega):=F(\omega, 0)=\mathcal{A}^{-1}\left(\beta(\lambda(\beta)+\beta) \omega\left(\beta^{2} \omega^{2}+|\nabla \omega|^{2}\right)^{p / 2-1}+\omega^{q}\right) ;
$$

hence any nontrivial fixed point for $\Phi$ would solve problem (3.3).

We have to verify the assumptions of Theorem 3.3. First of all, the compactness of $F(\omega, t)$ : If we set $F(\omega, t)=\phi$, then it means that $\phi \in W_{0}^{1, p}(S)$ satisfies

$$
\begin{aligned}
-\operatorname{div}_{g}\left(\left(\beta^{2} \phi^{2}+|\nabla \phi|^{2}\right)^{p / 2-1} \nabla \phi\right)+\beta^{2} \phi\left(\beta^{2} \phi^{2}+|\nabla \phi|^{2}\right)^{p / 2-1} & \\
& =\left(\beta(\lambda(\beta)+\beta+t) \omega\left(\beta^{2} \omega^{2}+|\nabla \omega|^{2}\right)^{p / 2-1}+(\omega+t)^{q}\right) .
\end{aligned}
$$

Thus, if we assume that $\omega$ belongs to a bounded set in $K \cap X$, the right-hand side of (3.6) is bounded in $C(\bar{S})$. Hence, by standard regularity estimates up to the boundary for $p$ Laplace type operators (see [13, Appendix] and [6], [19]), $\phi$ remains bounded in $C^{1, \alpha}(\bar{S})$ and therefore relatively compact in $C^{1}(\bar{S})$. It remains to show that conditions (i)-(iii) of Theorem 3.3 hold.

Step 1: Condition (i) holds. Suppose for contradiction that there exists a sequence $\left\{s_{n}\right\}$ in $[0,1]$ such that for any $n \in \mathbb{N}$ the problem

$$
\left\{\begin{array}{l}
-\operatorname{div}_{g}\left(\left(\beta^{2} \omega^{2}+|\nabla \omega|^{2}\right)^{p / 2-1} \nabla \omega\right)+\beta^{2}\left(\beta^{2} \omega^{2}+|\nabla \omega|^{2}\right)^{p / 2-1} \omega \\
=s^{p-1} \beta(\lambda(\beta)+\beta)\left(\beta^{2} \omega^{2}+|\nabla \omega|^{2}\right)^{p / 2-1} \omega+s_{n}^{p-1} \omega^{q} \text { in } S, \\
\omega=0 \text { on } \partial S,
\end{array}\right.
$$

admits a positive solution $\omega_{n}$, and that

$$
\left\|\omega_{n}\right\|_{X} \rightarrow 0 \quad \text { as } n \rightarrow \infty .
$$

Set $w_{n}=\omega_{n} /\left\|\omega_{n}\right\| ;$ then $w_{n}$ solves

$$
\left\{\begin{array}{l}
-\operatorname{div}_{g}\left(\left(\beta^{2} w_{n}^{2}+\left|\nabla w_{n}\right|^{2}\right)^{p / 2-1} \nabla w_{n}\right)+\beta^{2} w_{n}\left(\beta^{2} w_{n}^{2}+\left|\nabla w_{n}\right|^{2}\right)^{p / 2-1} \\
=s_{n}^{p-1} \beta(\lambda(\beta)+\beta)\left(\beta^{2} w_{n}^{2}+\left|\nabla w_{n}\right|^{2}\right)^{p / 2-1} w_{n}+s_{n}^{p-1} w_{n}^{q}\left\|w_{n}\right\|_{X}^{q-(p-1)} \\
w_{n}=0 \quad \text { on } \partial S .
\end{array}\right.
$$

Up to subsequences, we assume that $s_{n} \rightarrow s$ for some $s \in[0,1]$. Using compactness arguments we deduce that $w_{n}$ will converge strongly in $C^{1}(\bar{S})$ to some positive function $w$ such that $\|w\|_{X}=1$ and

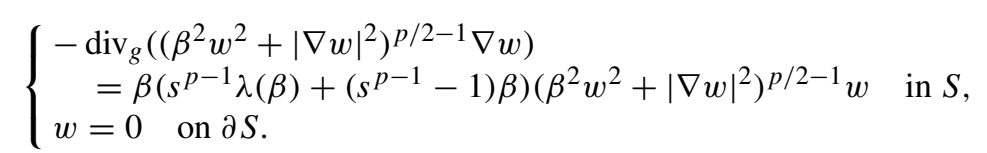

Using Theorem 3.1, we derive $\Lambda_{\beta}=s^{p-1} \lambda(\beta)+\left(s^{p-1}-1\right) \beta$. Since $\beta<\beta_{S}$, we have $\lambda(\beta)<\Lambda_{\beta}$ by (3.2). Therefore, as $s \leq 1$, we get

$$
s^{p-1} \lambda(\beta)+\left(s^{p-1}-1\right) \beta \leq s^{p-1} \lambda(\beta)<\Lambda_{\beta},
$$


which is a contradiction. Consequently, there exists $R_{1}>0$ such that for any $s \in[0,1]$, we have $\omega \neq s \Phi(\omega)$ for any $\omega$ such that $\|\omega\|_{X}=R_{1}$.

Step 2: Condition (ii) holds. Consider the first eigenvalue $\lambda_{1, \beta}$ associated with the operator $\mathcal{A}$, i.e.

$$
\lambda_{1, \beta}=\min \left\{\int_{S}\left(\beta^{2} \omega^{2}+|\nabla \omega|^{2}\right)^{p / 2} d v_{g}: \omega \in W_{0}^{1, p}(S), \int_{S}|\omega|^{p} d v_{g}=1\right\} .
$$

Note that for $t$ large enough, we have $\lambda(\beta)+\beta+t \geq 0$, hence, using that $q>p-1$, we can find $T>0$ such that

$$
\beta(\lambda(\beta)+\beta+t) \omega\left(\beta^{2} \omega^{2}+|\nabla \omega|^{2}\right)^{p / 2-1}+(\omega+t)^{q} \geq\left(\lambda_{1}+\delta\right) \omega^{p-1} \quad \forall t \geq T, \forall \omega \geq 0 .
$$

Therefore, if $t \geq T$ and $F(\omega, t)=\omega$ we deduce that $\omega \neq 0$ and satisfies

$$
\left\{\begin{array}{l}
-\operatorname{div}_{g}\left(\left(\beta^{2} \omega^{2}+|\nabla \omega|^{2}\right)^{p / 2-1} \nabla \omega\right)+\beta^{2} \omega\left(\beta^{2} \omega^{2}+|\nabla \omega|^{2}\right)^{p / 2-1} \geq\left(\lambda_{1, \beta}+\delta\right) \omega^{p-1} \text { in } S, \\
\omega=0 \text { on } \partial S .
\end{array}\right.
$$

The existence of a positive supersolution with $\lambda_{1, \beta}+\delta$ would make it possible to construct a positive solution as well. But since $\lambda_{1, \beta}$ is an isolated eigenvalue (see Appendix) this yields a contradiction. Therefore, for $t \geq T$ the equation $F(\omega, t)=\omega$ has no solution at all. Note that $T$ only depends on $\lambda_{1}, \beta$.

Step 3: Condition (iii) holds. Since we proved that (ii) holds independently of the choice of $R_{2}$, it is enough to show that (iii) holds for every $t \leq T$.

This is done if we have the existence of universal a priori estimates, i.e. if we can prove the existence of a constant $R_{2}$ such that for any $t \leq T$ every positive solution of

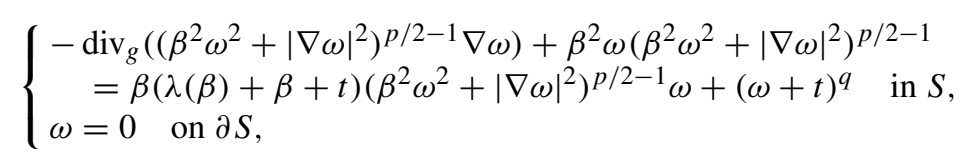

satisfies $\|\omega\|<R_{2}$.

The crucial step is to prove that there exist universal a priori estimates for the $L^{\infty}$ norm (a bound for the $W_{0}^{1, p}$-norm would follow immediately, and then a bound in $X$ from regularity theory). A standard procedure is to reach this result reasoning by contradiction and using a blow-up argument. Indeed, if a universal bound does not exist, there exist a sequence of solutions $\omega_{n}$ and $t_{n} \leq T$ such that

$$
\left\|\omega_{n}\right\|_{\infty} \rightarrow \infty .
$$

Let $\sigma_{n}$ be the (local coordinates of) maximum points of $\omega_{n}$; up to subsequences, we have $\sigma_{n} \rightarrow \sigma_{0} \in \bar{S}$. Setting $M_{n}=\left\|\omega_{n}\right\|_{\infty}^{-(q-(p-1)) / p}$, define

$$
v_{n}(y)=\frac{\omega_{n}\left(\sigma_{n}+M_{n} y\right)}{\left\|\omega_{n}\right\|_{\infty}}=M_{n}^{p /(q-(p-1))} \omega_{n}\left(\sigma_{n}+M_{n} y\right) .
$$


Then $v_{n}$ is a sequence of uniformly bounded solutions, which will be locally compact in the $C^{1}$-topology. Rescaling the equation and passing to the limit in $n$ we find that the limit function $v$ is positive and satisfies the equation

$$
-\Delta_{p} v=c_{0} v^{q}
$$

for some constant $c_{0}$ (coming from the local expression of the Laplace-Beltrami operator). Depending on whether $\sigma_{0} \in S$ or $\sigma_{0} \in \partial S$, the equation holds either in $\mathbb{R}^{d}$ or in the half-space $\mathbb{R}_{+}^{d}$, where $d=N-1$, in which case $v$ vanishes on $\partial \mathbb{R}_{+}^{d}$. Since $p-1<q<q_{c}$, this contradicts either Theorem 3.4, or Theorem 3.5, because, by construction, we have $v(0)=1$.

Remark. In the case $p=2$, existence is proved in [3] using a standard variational method. It is also proved that, if $(M, g)=\left(S^{d}, g_{0}\right)$ (the standard sphere), and if $S$ is a spherical cap with center $a$, then any positive solution of

$$
\left\{\begin{array}{l}
\left.\Delta^{\prime} \omega+\beta(\beta+1-d)\right) \omega+\omega^{q}=0 \text { in } S \\
\omega=0 \text { on } \partial S
\end{array}\right.
$$

depends only on the angle $\theta$ from $a$. Furthermore, uniqueness is proved by a delicate analysis of the nonautonomous second order O.D.E. satisfied by $\omega$. In the case $p \neq 2$ and assuming always that $S$ is a spherical cap of $\left(S^{d}, g_{0}\right)$, it is still possible to construct a radial (i.e. depending only on $\theta$ ) positive solution of (3.3): it suffices to restrict the functional analysis framework to radial functions. However, there are two interesting open questions the answer to which would be important:

(i) Are all positive solutions of (3.3) radial?

(ii) Is there uniqueness of positive radial solutions of (3.3)?

\section{Existence for the absorption problem}

Let us now consider the absorption problem, i.e. (1.8) with $\epsilon=-1$. We give an existence result which extends the previous ones obtained in [20], with a simpler proof.

Theorem 4.1. Assume $0<p-1<q$. Then for any $\beta>\beta_{S}$, there exists a unique positive function $\omega \in C(\bar{S}) \cap C^{2}(S)$ satisfying

$$
\left\{\begin{array}{l}
-\operatorname{div}_{g}\left(\left(\beta^{2} \omega^{2}+|\nabla \omega|^{2}\right)^{p / 2-1} \nabla \omega\right)=\beta \lambda(\beta)\left(\beta^{2} \omega^{2}+|\nabla \omega|^{2}\right)^{p / 2-1} \omega-\omega^{q} \text { in } S, \\
\omega=0 \text { on } \partial S,
\end{array}\right.
$$

where $\lambda(\beta)=\beta(p-1)+p-d-1$.

To prove Theorem 4.1, we will need the following lemma.

Lemma 4.1. For $\beta>0$ and $p>1$, let $\Lambda_{\beta}$ and $\beta_{S}$ be defined by Theorem 3.1. Then both $\Lambda_{\beta}$ and $\beta_{S}$ are continuous functions of $p$, varying in $(1, \infty)$. 
Proof. By Theorem 3.1, $\Lambda_{\beta}$ is uniquely defined for any fixed $p>1$. To emphasize the dependence of $\Lambda_{\beta}$ on $p$, let us denote it now by $\Lambda_{\beta, p}$. The continuity of $\Lambda_{\beta, p}$ with respect to $p$ can be proved in the same way as we proved (see Proposition 2.4 in [13]) the continuity of $\Lambda_{\beta, p}$ with respect to $\beta$. Thus, we only sketch the argument, which relies on the construction itself of $\Lambda_{\beta, p}$. Indeed, we proved in [13] that $\Lambda_{\beta, p}$ is the unique constant such that there exists a function $v \in C^{2}(S)$ satisfying

$$
\left\{\begin{array}{l}
-\Delta_{g} v-(p-2) \frac{D^{2} v \nabla v \cdot \nabla v}{1+|\nabla v|^{2}}+\beta(p-1)|\nabla v|^{2}=-\Lambda_{\beta, p} \quad \text { in } S, \\
\lim _{\sigma \rightarrow \partial S} v(\sigma)=\infty
\end{array}\right.
$$

If we normalize $v$ by setting, for example, $v\left(\sigma_{0}\right)=0$ for some $\sigma_{0} \in S$, then $v$ is unique. Moreover $v \in C^{2}(S)$ and $v$ satisfies estimates in $W_{\text {loc }}^{1, \infty}(S)$ which are uniform as $\beta \in$ $(0, \infty)$ and $p \in(1, \infty)$ vary in compact sets. It is also easy to check (see [13]) that $\Lambda_{\beta, p}$ remains bounded whenever $\beta$ varies in a compact subset of $(0, \infty)$ and $p$ vary in a compact subset of $(1, \infty)$. The estimates obtained on $v$ and $\nabla v$ imply that, whenever $\beta_{n}$ or $p_{n}$ are convergent sequences, the sequence of the corresponding solutions $v_{n}$ of (4.2) (such that $v_{n}\left(\sigma_{0}\right)=0$ ) is relatively compact (locally uniformly in $C^{1}$ ). The equation (4.2) turns out then to be stable (including the boundary estimates); finally, the uniqueness property of $\Lambda_{\beta, p}$, and of the associated (normalized) solution $v$, implies the continuity of $\Lambda_{\beta, p}$ with respect to both $\beta$ and $p$.

Let now $\beta_{S, p}$ be the spectral exponent defined by the equation

$$
\Lambda_{\beta, p}=\beta(p-1)+p-d-1 .
$$

First of all note that when $p$ lies in a compact set in $(1, \infty)$, then necessarily $\beta_{S, p}$ is bounded. Indeed, since $\Lambda_{\beta, p} \leq \Lambda_{1, p}$ whenever $\beta \geq 1$, we have

$$
\beta_{S}(p-1)+p-d-1 \leq \Lambda_{1, p} \quad \text { if } \beta_{S} \geq 1,
$$

so that

$$
\beta_{S} \leq 1+\frac{1}{p-1}\left(\Lambda_{1, p}-(p-d-1)\right) .
$$

Therefore, if $p$ belongs to a compact set in $(1, \infty)$, then $\beta_{S}$ remains also in a bounded set. Now, if $p_{n} \rightarrow p_{0}$, setting $\beta_{n}=\beta_{S, p_{n}}$, we see that $\beta_{n}$ is bounded and, up to subsequences, it is convergent to some $\beta_{0}$. From (4.3), we deduce that $\Lambda_{\beta_{n}, p_{n}}$ is bounded, which implies that $\beta_{n}$ cannot converge to zero, hence $\beta_{0}>0$. Then, using the continuity of $\Lambda_{\beta, p}$, we can pass to the limit in (4.3) and we deduce that $\beta_{0}$ is the spectral exponent with $p=p_{0}$, i.e. $\beta_{0}=\beta_{S, p_{0}}$. This proves that $\beta_{S, p}$ is continuous with respect to $p$.

We are now ready to prove Theorem 4.1.

\section{Proof of Theorem 4.1}

Step 1: construction of a solution. We use similar ideas to the proof of Theorem 3.2, i.e. a topological degree argument. On the Banach space $X=C_{0}^{1}(\bar{S})$ (endowed with its natural 
norm) with positive cone $K$, we set

$$
\begin{aligned}
& \mathcal{B}(\omega)=-\operatorname{div}_{g}\left(\left(\beta^{2} \omega^{2}+|\nabla \omega|^{2}\right)^{p / 2-1} \nabla \omega\right)+\beta^{2}\left(\beta^{2} \omega^{2}+|\nabla \omega|^{2}\right)^{p / 2-1} \omega+|\omega|^{q-1} \omega, \\
& \Psi(\omega)=\mathcal{B}^{-1}\left(\beta(\lambda(\beta)+\beta)\left(\beta^{2} \omega^{2}+|\nabla \omega|^{2}\right)^{p / 2-1} \omega_{+}\right) .
\end{aligned}
$$

Clearly, $\Psi(w)=w$ implies that $w \geq 0$ and solves (4.1). Then, it is enough to prove the existence of a nontrivial fixed point for $\Psi$. Observe that, as in Theorem 3.2, $\Psi$ is a continuous compact operator in $X$ thanks to the $C^{1, \alpha}$ estimates for $p$-Laplace operators, and $\Psi(K) \subset K$.

We now wish to compute the degree of $I-\Psi$. First of all we consider, if $R$ is sufficiently large, $\operatorname{deg}\left(I-\Psi, B_{R}^{+}, 0\right)$ where $B_{R}^{+}=B_{R} \cap K$ for $t \in[0,1]$. To this end, define $\Psi^{*}(\omega, t)=t \Psi(\omega)$. Then $\Psi^{*}$ is a compact map on $X \times[0,1]$ and if $\Psi^{*}(\omega, t)=\omega$, we have

$$
\begin{array}{r}
-\operatorname{div}_{g}\left(\left(\beta^{2} \omega^{2}+|\nabla \omega|^{2}\right)^{p / 2-1} \nabla \omega\right)+\beta^{2}\left(\beta^{2} \omega^{2}+|\nabla \omega|^{2}\right)^{p / 2-1} \omega+\frac{1}{t^{q-(p-1)}} \omega^{q} \\
=t^{p-1} \beta(\lambda(\beta)+\beta)\left(\beta^{2} \omega^{2}+|\nabla \omega|^{2}\right)^{p / 2-1} \omega
\end{array}
$$

We get, by the maximum principle,

$$
\left\|\frac{\omega}{t}\right\|_{\infty}^{q-(p-1)} \leq t^{p-1} \beta^{p-1}(\lambda(\beta)+\beta) \leq \beta^{p-1}(\lambda(\beta)+\beta) .
$$

Since $t \leq 1$, we deduce in particular that $\|\omega\|_{\infty}$ is bounded independently of $t$. Then, we have

$$
\frac{1}{t^{q-(p-1)}} \omega^{q} \leq\left\|\frac{\omega}{t}\right\|_{\infty}^{q-(p-1)}\|\omega\|_{\infty}^{p-1} \leq C\|\omega\|_{\infty}^{p-1} \leq C .
$$

Multiplying by $\omega$ we obtain a similar bound for $\|\omega\|_{W_{0}^{1, p}(S)}$, and the regularity theory for $p$-Laplace type equations yields a further estimate on $\|\nabla \omega\|_{\infty}$. Therefore, we conclude that there exists a constant $M$, independent of $t \in[0,1]$, such that $t \Psi(\omega)=\omega$ implies $\|\omega\|_{X} \leq M$. As a consequence, if $R$ is sufficiently large we have $t \Psi(\omega) \neq \omega$ on $\partial B_{R}$. We deduce that $\operatorname{deg}\left(I-t \Psi, B_{R}^{+}, 0\right)$ is constant. Therefore

$$
\operatorname{deg}\left(I-\Psi, B_{R}^{+}, 0\right)=\operatorname{deg}\left(I-t \Psi, B_{R}^{+}, 0\right)=\operatorname{deg}\left(I, B_{R}^{+}, 0\right)=1 .
$$

Next, we compute $\operatorname{deg}\left(I-\Psi, B_{r}^{+}, 0\right)$ for small $r$. We set

$$
\begin{aligned}
\mathcal{B}_{t}(\omega) & =-\operatorname{div}_{g}\left(\left(\beta^{2} \omega^{2}+|\nabla \omega|^{2}\right)^{p / 2-1} \nabla \omega\right)+\beta^{2}\left(\beta^{2} \omega^{2}+|\nabla \omega|^{2}\right)^{p / 2-1} \omega+t|\omega|^{q-1} \omega, \\
F(\omega, t) & =\mathcal{B}_{t}^{-1}\left(\beta(\lambda(\beta)+\beta) \omega_{+}\left(\beta^{2} \omega^{2}+|\nabla \omega|^{2}\right)^{p / 2-1}\right) .
\end{aligned}
$$

Again, we have $\Psi(\cdot)=F(\cdot, 1)$. We claim that there exists a small $r>0$ such that $F(\omega, t) \neq \omega$ for every $t \in[0,1]$ and $\omega \in \partial B_{r}$. Indeed, if this were not true there would 
exist a nonnegative sequence $\omega_{n}$ such that $0 \neq\left\|\omega_{n}\right\| \rightarrow 0$, and $t_{n} \in[0,1]$ such that $F\left(\omega_{n}, t_{n}\right)=\omega_{n}$, which means that

$$
\begin{aligned}
-\operatorname{div}_{g}\left(\left(\beta^{2} \omega_{n}^{2}+\left|\nabla \omega_{n}\right|^{2}\right)^{p / 2-1} \nabla \omega_{n}\right)+ & \beta^{2}\left(\beta^{2} \omega_{n}^{2}+\left|\nabla \omega_{n}\right|^{2}\right)^{p / 2-1} \omega_{n}+t_{n} \omega_{n}^{q} \\
& =\beta(\lambda(\beta)+\beta) \omega_{n}\left(\beta^{2} \omega_{n}^{2}+\left|\nabla \omega_{n}\right|^{2}\right)^{p / 2-1} .
\end{aligned}
$$

Dividing by $\left\|\omega_{n}\right\|^{p-1}$ and letting $n \rightarrow \infty$, we find that $\omega_{n} /\left\|\omega_{n}\right\|$ would converge to some function $\hat{\omega}$ such that $\hat{\omega} \geq 0,\|\hat{\omega}\|=1$ and

$$
\begin{aligned}
-\operatorname{div}_{g}\left(\left(\beta^{2} \hat{\omega}^{2}+|\nabla \hat{\omega}|^{2}\right)^{p / 2-1} \nabla \hat{\omega}\right)+\beta^{2}\left(\beta^{2} \hat{\omega}^{2}+|\nabla \hat{\omega}|^{2}\right)^{p / 2-1} \hat{\omega} & \\
& =\beta(\lambda(\beta)+\beta) \hat{\omega}\left(\beta^{2} \hat{\omega}^{2}+|\nabla \hat{\omega}|^{2}\right)^{p / 2-1} .
\end{aligned}
$$

By Theorem 3.1 this means that $\lambda(\beta)=\Lambda_{\beta}$, which is not possible since $\lambda(\beta)>\Lambda_{\beta}$ because $\beta>\beta_{S}$ (see Remark 3.1). We conclude that $F(\omega, t) \neq \omega$ for every $t \in[0,1]$ and $\omega \in \partial B_{r}$ provided $r$ is sufficiently small. We deduce that $\operatorname{deg}\left(I-F(\cdot, t), B_{r}, 0\right)$ is constant and in particular

$$
\operatorname{deg}\left(I-\Psi, B_{r}^{+}, 0\right)=\operatorname{deg}\left(I-F(\cdot, 0), B_{r}^{+}, 0\right) .
$$

In order to compute this degree, we perform a homotopy acting on $p$ and $\beta$ by setting $p_{t}=2 t+(1-t) p$ and by taking $\beta_{t}$ so that $t \mapsto \beta_{t}$ is continuous on $[0,1], \beta_{0}=\beta$, $\beta_{t}>\beta_{S, p_{t}}$ for every $t \in[0,1]$ (where $\beta_{S, p_{t}}$ is the spectral exponent for $S$ with $p=p_{t}$ ) and $\beta_{1}>0$ is large enough. It follows from Lemma 4.1 that $\beta_{S, p_{t}}$ is a continuous function of $t$ and remains bounded as $t \in[0,1]$. Therefore, a similar choice of a function $\beta_{t}$ is possible. In the space $C_{0}^{1}(\bar{S})$ we define the mapping $\mathrm{C}_{t}$ by

$$
\mathcal{C}_{t}(\omega)=-\operatorname{div}_{g}\left(\left(\beta_{t}^{2} \omega^{2}+|\nabla \omega|^{2}\right)^{p_{t} / 2-1} \nabla \omega\right)+\beta_{t}^{2}\left(\beta_{t}^{2} \omega^{2}+|\nabla \omega|^{2}\right)^{p_{t} / 2-1} \omega .
$$

We set

$$
\tilde{F}(\omega, t)=\mathcal{C}_{t}^{-1}\left(\beta_{t}\left(\lambda\left(\beta_{t}\right)+\beta_{t}\right)\left(\beta_{t}^{2} \omega^{2}+|\nabla \omega|^{2}\right)^{p_{t} / 2-1} \omega\right) .
$$

Combining Tolksdorf's construction [19] which shows the uniformity with respect to $p_{t}$ of the $C^{1, \alpha}$ estimates (with $\alpha=\alpha_{t} \in(0,1)$ ), with the perturbation method of [13, Th. A1], we deduce that $(\omega, t) \mapsto \tilde{F}(\omega, t)$ is compact in $C_{0}^{1}(\bar{S}) \times[0,1]$. Since $\beta_{t}>\beta_{S, p_{t}}$, clearly $I-\tilde{F}(\cdot, t)$ does not vanish on $\|\omega\|_{X}=r$ for any $r>0$, which implies that

$$
\operatorname{deg}\left(I-\Psi, B_{r}^{+}, 0\right)=\operatorname{deg}\left(I-\tilde{F}(\cdot, 0), B_{r}^{+}, 0\right)=\operatorname{deg}\left(I-\tilde{F}(\cdot, 1), B_{r}^{+}, 0\right) .
$$

But

$$
I-\tilde{F}(\cdot, 1)=I-\beta_{1}\left(\lambda\left(\beta_{1}\right)+\beta_{1}\right)\left(-\Delta_{g}+\beta_{1}^{2}\right)^{-1} .
$$

Since $-\Delta_{g}$ has only one eigenvalue in $S$ with positive eigenfunction and multiplicity one, choosing $\beta_{1}$ so large that $\lambda\left(\beta_{1}\right) \beta_{1}>\lambda_{1}(S)$ it follows that

$$
\operatorname{deg}\left(I-\tilde{F}(\cdot, 1), B_{r}^{+}, 0\right)=-1=\operatorname{deg}\left(I-\Psi, B_{r}^{+}, 0\right) .
$$


To conclude, since

$$
\operatorname{deg}\left(I-\Psi, B_{R}^{+} \backslash \bar{B}_{r}^{+}, 0\right)=\operatorname{deg}\left(I-\Psi, B_{R}^{+}, 0\right)-\operatorname{deg}\left(I-\Psi, B_{r}^{+}, 0\right) \neq 0
$$

we deduce the existence of some $\omega$ such that $r<\|\omega\|<R$ which is a solution of (4.1).

Step 2: uniqueness. If $\omega$ is any positive solution, then $\beta^{2} \omega^{2}+\left|\nabla \omega^{2}\right|$ is positive in $\bar{S}$. This is obvious in $S$ and it is a consequence of the Hopf boundary lemma on $\partial S$. Let $\bar{\omega}$ and $\omega$ be two positive solutions. Either the two functions are ordered or their graphs intersect. Since all the solutions are positive in $S$ and satisfy the Hopf boundary lemma, we can define

$$
\theta:=\inf \{s \geq 1: s \omega \geq \bar{\omega}\}
$$

and denote $\omega^{*}:=\theta \omega$. Either the graphs of $\bar{\omega}$ and $\omega^{*}:=\theta \omega$ are tangent at some interior point $\alpha \in S$, or $\omega^{*}>\bar{\omega}$ in $S$ and there exists $\alpha \in \partial S$ such that $\bar{\omega}_{v}(\alpha)=\omega_{v}^{*}(\alpha)<0$. We put $w=\bar{\omega}-\omega^{*}$ and use local coordinates $\left(\sigma_{1}, \ldots, \sigma_{d}\right)$ on $M$ near $\alpha$. We denote by $g=\left(g_{i j}\right)$ the metric tensor on $M$ and $g^{j k}$ its contravariant components. Then, for any $\varphi \in C^{1}(S)$,

$$
|\nabla \varphi|^{2}=\sum_{j, k} g^{j k} \frac{\partial \varphi}{\partial \sigma_{j}} \frac{\partial \varphi}{\partial \sigma_{k}}=\langle\nabla \varphi, \nabla \varphi\rangle_{g}
$$

If $X=\left(X^{1}, \ldots, X^{d}\right) \in C^{1}(T M)$ is a vector field, if we lower indices by setting $X^{\ell}=\sum_{i} g^{\ell i} X_{i}$, then

$$
\operatorname{div}_{g} X=\frac{1}{\sqrt{|g|}} \sum_{\ell} \frac{\partial}{\partial \sigma_{\ell}}\left(\sqrt{|g|} X^{\ell}\right)=\frac{1}{\sqrt{|g|}} \sum_{\ell, i} \frac{\partial}{\partial \sigma_{\ell}}\left(\sqrt{|g|} g^{\ell i} X_{i}\right)
$$

By the mean value theorem applied to

$$
t \mapsto \Phi(t)=\left(\beta^{2}\left(\omega^{*}+t w\right)^{2}+\left|\nabla\left(\omega^{*}+t w\right)\right|^{2}\right)^{p / 2-1}\left(\omega^{*}+t w\right), \quad t \in[0,1],
$$

we have, for some $t \in(0,1)$,

$$
\left(\beta^{2} \bar{\omega}^{2}+|\nabla \bar{\omega}|^{2}\right)^{p / 2-1} \bar{\omega}-\left(\beta^{2} \omega^{* 2}+\left|\nabla \omega^{*}\right|^{2}\right)^{p / 2-1} \omega^{*}=\sum_{j} a_{j} \frac{\partial w}{\partial \sigma_{j}}+b w,
$$

where

$$
b=\left(\beta^{2}\left(\omega^{*}+t w\right)^{2}+\left|\nabla\left(\omega^{*}+t w\right)\right|^{2}\right)^{p / 2-2}\left((p-1) \beta^{2}\left(\omega^{*}+t w\right)^{2}+\left|\nabla\left(\omega^{*}+t w\right)\right|^{2}\right)
$$

and

$$
a_{j}=(p-2)\left(\beta^{2}\left(\omega^{*}+t w\right)^{2}+\left|\nabla\left(\omega^{*}+t w\right)\right|^{2}\right)^{p / 2-2}\left(\omega^{*}+t w\right) \sum_{k} g^{j k} \frac{\partial\left(\omega^{*}+t w\right)}{\partial \sigma_{k}} .
$$

Considering now

$$
t \mapsto \Phi_{i}(t)=\left(\beta^{2}\left(\omega^{*}+t w\right)^{2}+\left|\nabla\left(\omega^{*}+t w\right)\right|^{2}\right)^{p / 2-1} \frac{\partial\left(\omega^{*}+t w\right)}{\partial \sigma_{i}}, \quad t \in[0,1],
$$


we see that there exists some $t_{i} \in(0,1)$ such that

$$
\left(\beta^{2} \bar{\omega}^{2}+|\nabla \bar{\omega}|^{2}\right)^{p / 2-1} \frac{\partial \bar{\omega}}{\partial \sigma_{i}}-\left(\beta^{2} \omega^{* 2}+\left|\nabla \omega^{*}\right|^{2}\right)^{p / 2-1} \frac{\partial \omega^{*}}{\partial \sigma_{i}}=\sum_{j} a_{i j} \frac{\partial w}{\partial \sigma_{j}}+b_{i} w,
$$

where

$$
b_{i}=(p-2)\left(\beta^{2}\left(\omega^{*}+t_{i} w\right)^{2}+\left|\nabla\left(\omega^{*}+t_{i} w\right)\right|^{2}\right)^{p / 2-2} \beta^{2}\left(\omega^{*}+t_{i} w\right) \frac{\partial\left(\omega^{*}+t_{i} w\right)}{\partial \sigma_{i}}
$$

and

$$
\begin{aligned}
a_{i j}= & (p-2)\left(\beta^{2}\left(\omega^{*}+t_{i} w\right)^{2}+\left|\nabla\left(\omega^{*}+t_{i} w\right)\right|^{2}\right)^{p / 2-2} \frac{\partial\left(\omega^{*}+t_{i} w\right)}{\partial \sigma_{i}} \sum_{k} g^{j k} \frac{\partial\left(\omega^{*}+t_{i} w\right)}{\partial \sigma_{k}} \\
& +\delta_{i}^{j}\left(\beta^{2}\left(\omega^{*}+t_{i} w\right)^{2}+\left|\nabla\left(\omega^{*}+t_{i} w\right)\right|^{2}\right)^{p / 2-1} .
\end{aligned}
$$

Set $P=\omega^{*}(\alpha)=\bar{\omega}(\alpha)$ and $Q=\nabla \omega^{*}(\alpha)=\nabla \bar{\omega}(\alpha)$. Then $P^{2}+|Q|^{2}>0$ and

$$
b_{i}(\alpha)=(p-2)\left(\beta^{2} P^{2}+|Q|^{2}\right)^{p / 2-2} \beta^{2} P Q_{i},
$$

and

$$
a_{i j}(\alpha)=\left(\beta^{2} P^{2}+|Q|^{2}\right)^{p / 2-2}\left(\delta_{i}^{j}\left(\beta^{2} P^{2}+|Q|^{2}\right)+(p-2) Q_{i} \sum_{k} g^{j k} Q_{k}\right) .
$$

Because $\omega^{*}$ is a supersolution for (4.1), the function $w$ satisfies

$$
-\frac{1}{\sqrt{|g|}} \sum_{\ell, j} \frac{\partial}{\partial \sigma_{\ell}}\left(A_{j \ell} \frac{\partial w}{\partial \sigma_{j}}\right)+\sum_{i} C_{i} \frac{\partial w}{\partial \sigma_{i}}+D w \leq 0
$$

where the $C_{i}$ and $D$ are continuous functions and

$$
A_{j \ell}=\sqrt{|g|} \sum_{i} g^{\ell i} a_{i j}
$$

The matrix $\left(a_{i j}\right)(\alpha)$ is symmetric and positive definite since it is the Hessian of

$$
x=\left(x_{1}, \ldots, x_{d}\right)=\frac{1}{p}\left(P^{2}+|x|^{2}\right)^{p / 2}=\frac{1}{p}\left(P^{2}+\sum_{j, k} g^{j k} x_{j} x_{k}\right)^{p / 2} .
$$

Therefore the matrix $\left(A_{j \ell}\right)$ keeps the same property in a neighborhood of $a$. Since $w$ is nonpositive and vanishes at some $a \in S$, or $w<0$ and $w_{\nu}=0$ at some boundary point, it follows from the strong maximum principle or the Hopf boundary lemma (see [14]) that $w \equiv 0$, i.e. $\theta \omega=\bar{\omega}$. This implies that actually $\theta=1$ and $\omega=\bar{\omega}$. 


\section{Appendix}

Here we prove the following result:

Theorem 5.1. Let $S$ be a subdomain of a complete d-dimensional Riemannian manifold $(M, g)$. If $\beta>0$ and $p>1$, then the first eigenvalue $\lambda_{1, \beta}$ of the operator $\omega \mapsto$ $-\operatorname{div}\left(\left(\beta^{2} \omega^{2}+|\nabla \omega|^{2}\right)^{p / 2-1} \nabla \omega\right)+\beta^{2} \omega\left(\beta^{2} \omega^{2}+|\nabla \omega|^{2}\right)^{p / 2-1}$ in $W_{0}^{1, p}(S)$ is isolated. Furthermore any corresponding eigenfunction has constant sign.

Proof. The proof is an adaptation of the original one due to Anane [1] and Lindqvist [10, 11] when $\beta=0$. We recall that

$$
\lambda_{1, \beta}=\inf \left\{\int_{S}\left(\beta^{2} \omega^{2}+|\nabla \omega|^{2}\right)^{p / 2} d v_{g}: \omega \in W_{0}^{1, p}(S), \int|\omega|^{p} d v_{g}=1\right\},
$$

and that there exists $\omega \in W_{0}^{1, p}(S) \cap C^{1, \alpha}(S)$ such that

$$
-\operatorname{div}\left(\left(\beta^{2} \omega^{2}+|\nabla \omega|^{2}\right)^{p / 2-1} \nabla \omega\right)+\beta^{2} \omega\left(\beta^{2} \omega^{2}+|\nabla \omega|^{2}\right)^{p / 2-1}=\lambda_{1, \beta}|\omega|^{p-2} \omega \quad \text { in } S .
$$

The function $|\omega|$ is also a minimizer for $\lambda_{1, \beta}$, thus it is a positive solution of (5.2). By the Harnack inequality [16], for any compact subset $K$ of $S$, there exists $C_{K}$ such that

$$
\frac{|\omega|\left(\sigma_{1}\right)}{|\omega|\left(\sigma_{2}\right)} \leq C_{K} \quad \forall \sigma_{i} \in K, i=1,2 .
$$

Thus any minimizer $\omega$ must keep a constant sign in $S$. If $\lambda_{1, \beta}$ is not isolated, there exists a decreasing sequence $\left\{\mu_{n}\right\}$ of real numbers converging to $\lambda_{1, \beta}$ and a sequence of functions $\omega_{n} \in W_{0}^{1, p}(S)$ satisfying

$\left.-\operatorname{div}\left(\beta^{2} \omega_{n}^{2}+\left|\nabla \omega_{n}\right|^{2}\right)^{p / 2-1} \nabla \omega_{n}\right)+\beta^{2} \omega_{n}\left(\beta^{2} \omega_{n}^{2}+\left|\nabla \omega_{n}\right|^{2}\right)^{p / 2-1}=\mu_{n}\left|\omega_{n}\right|^{p-2} \omega_{n} \quad$ in $S$

such that $\left\|\omega_{n}\right\|_{L^{p}(S)}=1$. By standard compactness and regularity results, we can assume that $\omega_{n} \rightarrow \bar{\omega}$ weakly in $W_{0}^{1, p}(S)$ and strongly in $L^{p}(S)$. Thus

$$
\int_{S}\left(\beta^{2} \bar{\omega}^{2}+|\nabla \bar{\omega}|^{2}\right)^{p / 2} d v_{g} \leq \liminf _{n \rightarrow \infty} \int_{S}\left(\beta^{2} \omega_{n}^{2}+\left|\nabla \omega_{n}\right|^{2}\right)^{p / 2} d v_{g}=\lambda_{1, \beta},
$$

which implies that $\bar{\omega}$ is an eigenfunction associated with $\lambda_{1, \beta}$.

We observe that $\omega_{n}$ cannot have constant sign. Indeed, if $\omega_{n}$ were positive in $\Omega$, we could proceed as in the proof of Theorem 4.1, Step 2; up to rescaling $\omega_{n}$, we could assume that $w=\omega-\omega_{n}$ is nonpositive, is not zero, and the graphs of $\omega$ and $\omega_{n}$ are tangent. In that case, using (5.2) and (5.3), we see that $w$ satisfies a nondegenerate elliptic equation (as in (4.5)), and we obtain a contradiction either by the strict maximum principle or by the Hopf lemma. Thus, any eigenfunction $\omega_{n}$ must change sign in $\Omega$. Set $S_{n}^{+}=\{\sigma \in S$ : $\left.\omega_{n}(\sigma)>0\right\}$ and $S_{n}^{-}=\left\{\sigma \in S: \omega_{n}(\sigma)<0\right\}$. Clearly, for $0<\theta<1$,

$$
\int_{S_{n}^{ \pm}}\left(\beta^{2} \omega_{n}^{2}+\left|\nabla \omega_{n}\right|^{2}\right)^{p / 2} d v_{g} \geq(1-\theta) \beta^{p} \int_{S_{n}^{ \pm}}\left|\omega_{n}\right|^{p} d v_{g}+\theta \int_{S_{n}^{ \pm}}\left|\nabla \omega_{n}\right|^{p} d v_{g} .
$$


It follows from (5.3), multiplying by $\omega_{n}^{+}$, that

$$
\int_{S_{n}^{+}}\left(\beta^{2} \omega_{n}^{2}+\left|\nabla \omega_{n}\right|^{2}\right)^{p / 2} d v_{g}=\mu_{n} \int_{S_{n}^{+}}\left|\omega_{n}\right|^{p} d v_{g}
$$

hence

$$
\mu_{n} \int_{S_{n}^{+}}\left|\omega_{n}\right|^{p} d v_{g} \geq(1-\theta) \beta^{p} \int_{S_{n}^{+}}\left|\omega_{n}\right|^{p} d v_{g}+\theta \int_{S_{n}^{+}}\left|\nabla \omega_{n}\right|^{p} d v_{g} .
$$

Since for some suitable $q>p$ (for example $q=p^{*}$ if $p<d$, or any $p<q<\infty$ if $p \geq d)$

$$
\int_{S_{n}^{+}}\left|\nabla \omega_{n}\right|^{p} d v_{g} \geq c(p, q)\left(\int_{S_{n}^{+}}\left|\omega_{n}\right|^{q} d v_{g}\right)^{p / q} \geq c(p, q)\left|S_{n}^{+}\right|^{(p-q) / q} \int_{S_{n}^{+}}\left|\omega_{n}\right|^{p} d v_{g}
$$

we obtain

$$
\mu_{n} \geq(1-\theta) \beta^{p}+\theta c(p, q)\left|S_{n}^{+}\right|^{(p-q) / q} .
$$

Similarly we get, multiplying (5.3) by $\omega_{n}^{-}$,

$$
\mu_{n} \geq(1-\theta) \beta^{p}+\theta c(p, q)\left|S_{n}^{-}\right|^{(p-q) / q} .
$$

It follows that the two sets

$$
S^{ \pm}=\limsup _{n \rightarrow \infty} S_{n}^{ \pm}
$$

have positive measure. Since $\bar{\omega} \geq 0$ on $S^{+}$and $\bar{\omega} \leq 0$ on $S^{-}$, we derive a contradiction with the fact that any eigenfunction corresponding to $\lambda_{1, \beta}$ has constant sign.

\section{References}

[1] Anane, A.: Simplicité et isolation de la première valeur propre du $p$-laplacien avec poids. C. R. Acad. Sci. Paris Sér. I Math. 305, 725-728 (1987) Zbl 0633.35061 MR 0920052

[2] Bidaut-Véron, M. F., Jazar, M., Véron, L.: Separable solutions of some quasilinear equations with source reaction J. Differential Equations 244, 274-308 (2008) Zbl 1136.35041 MR 2376199

[3] Bidaut-Véron, M. F., Ponce, A., Véron, L.: Isolated boundary singularities of semilinear elliptic equations. Calc. Var. Partial Differential Equations 40, 183-221 (2011) Zbl 1215.35075 MR 2745200

[4] Borghol, R., Véron, L.: Boundary singularities of solutions of $N$-harmonic equations with absorption. J. Funct. Anal. 241, 611-637 (2006) Zbl pre05116349 MR 2271931

[5] de Figueiredo, D., Lions, P.-L., Nussbaum, R.: A priori estimates and existence of positive solutions of semilinear elliptic equations. J. Math. Pures Appl. 61, 41-63 (1982) Zbl 0452.35030 MR 0664341

[6] DiBenedetto, E.: $C^{1+\alpha}$ local regularity of weak solutions of degenerate elliptic equations. Nonlinear Anal. 7, 827-850 (1983) Zbl 0539.35027 MR 0709038

[7] Gmira, A., Véron, L.: Boundary singularities of solutions of some nonlinear elliptic equations. Duke Math. J. 60, 271-324 (1991) Zbl 0766.35015 MR 1136377 
[8] Huentutripay, J., Jazar, M., Véron, L.: A dynamical system approach to the construction of singular solutions of some degenerate elliptic equations. J. Differential Equations 195, 175193 (2003) Zbl 1101.35032 MR 2019247

[9] Krasnosel'skiř, M. A.: Positive Solutions of Operator Equations. Noordhoff, Groningen (1964) MR 0181881

[10] Lindqvist, P.: On the equation $\operatorname{div}\left(|\nabla u|^{p-2} \nabla u\right)+\lambda|u|^{p-2} u=0$. Proc. Amer. Math. Soc. 109, 157-164 (1990) Zbl 0714.35029 MR 1007505

[11] Lindqvist, P.: On a nonlinear eigenvalue problem. In: Fall School in Analysis Jyväskylä, Report 68 Univ. Jyväskylä, 33-54 (1995) Zbl 0838.35094 MR 1351043

[12] Lions, J.-L.: Quelques méthodes de résolution des problèmes aux limites non linéaires. Dunod and Gauthier-Villars, Paris (1969) Zbl 0189.40603 MR 0259693

[13] Porretta, A., Véron, L.: Separable $p$-harmonic functions in a cone and related quasilinear equations on manifolds. J. Eur. Math. Soc. 11, 1285-1305 (2009) Zbl 1203.35101 MR 2557136

[14] Protter, M., Weinberger, H.: Maximum Principles in Differential Equations. Prentice-Hall (1967) Zbl 0153.13602 MR 0219861

[15] Quaas, A., Sirakov, B.: Existence results for nonproper elliptic equations involving the Pucci operator. Comm. Partial Differential Equations 31, 987-1003 (2006) Zbl 1237.35056 MR 2254600

[16] Serrin, J.: Local behavior of solutions of quasi-linear equations. Acta Math. 111, 247-302 (1964) Zbl 0128.09101 MR 0170096

[17] Serrin, J., Zou, H. H.: Cauchy-Liouville and universal boundedness theorems for quasilinear elliptic equations and inequalities. Acta Math. 189, 79-142 (2002) Zbl 1059.35040 MR 1946918

[18] Tolksdorf, P.: On the Dirichlet problem for quasilinear equations in domains with conical boundary points. Comm. Partial Differential Equations 8, 773-817 (1983) Zbl 0515.35024 MR 0700735

[19] Tolksdorf, P.: Regularity for a more general class of quasilinear elliptic equations. J. Differential Equations 51, 126-150 (1984) Zbl 0488.35017 MR 0727034

[20] Véron, L.: Some existence and uniqueness results for solution of some quasilinear elliptic equations on compact Riemannian manifolds. In: Colloq. Math. Soc. János Bolyai 62, NorthHolland, 317-352 (1991) Zbl 0822.58052 MR 1468764

[21] Véron, L.: Singularities of Solutions of Second Order Quasilinear Elliptic Equations. Pitman Res. Notes in Math. 353, Addison-Wesley and Longman (1996) Zbl 0858.35018 MR 1424468

[22] Véron, L.: Singular $p$-harmonic functions and related quasilinear equations on manifolds. Electron. J. Differential Equations Conf. 8, 133-154 (2002) Zbl 1114.35319 MR 1990300

[23] Zou, H. H.: A priori estimates and existence for quasi-linear elliptic equations. Calc. Var. Partial Differential Equations 34, 417-437 (2008) Z Zbl 1169.35336 MR 2438741 